\title{
A Comparative Study of Quality of Family, Social Relationships and Type D Personality in Cardiovascular Patients and Non-Patients
}

\author{
Shervin Ziabakhsh Tabary¹, Mohammad Rezaii ${ }^{2}$, Ziba Javari Nia ${ }^{3}$, Pegah Goodarzy ${ }^{4}$, \\ Maryam Izanloo ${ }^{*}$ \\ ${ }^{1}$ Department of Cardiac Surgery, Mazandaran Heart Center, Mazandaran University of Medical Sciences, \\ Sari, Iran \\ ${ }^{2}$ Allameh Tabatabai University, Tehran, Iran \\ ${ }^{3}$ Health Management and Economics Research Center, Iran University of Medical Sciences, Tehran, Iran \\ ${ }^{4}$ Hospital Management Research Center, Iran University of Medical Sciences, Tehran, Iran \\ ${ }^{5}$ Research Center for Health Services Management, Institute for Futures Studies in Health, Kerman University \\ of Medical Sciences, Kerman, Iran \\ Email: maryame65@yahoo.com
}

Received 30 June 2014; revised 17 August 2014; accepted 31 August 2014

Copyright (C) 2014 by authors and Scientific Research Publishing Inc.

This work is licensed under the Creative Commons Attribution International License (CC BY).

http://creativecommons.org/licenses/by/4.0/

c) (i) Open Access

\section{Abstract}

This study aims to compare the quality of family and social relationships and Type $D$ personality in cardiovascular patients with normal people. In this research, 300 individuals $(n=150$ male, 150 female) are divided in two groups of patient $(n=150)$ and non-patients $(n=150)$ were selected as samples from the author's offices (both private and his office in Mazandaran Heart Center). They attended in this study filling the questionnaires of "Pierce Quality relations" and "Personality Inventory (DS14)". In order to analyze data, First observations were described by the help of descriptive statistics methods including tables, graphs, and indices of central tendency and distribution. Then, observations were described by inferential statistics methods such as univariate and multivariate analysis of variance and then were analyzed by SPSS. Out of the 300 participants, 108 patients (36\%) had the mean age of 52 to 63 years old and 30 patients $(10 \%)$ were 30 to 40 years old. This study showed that there is a significant difference between cardiovascular patients and control group in terms of quality of family and social relationships and Type $D$ personality $(P$ $<0.01$ ). Cardiovascular patients have lower quality of family and social relationships, social support, as well as lower depth of relationships, and also higher relationship conflict than normal 
people. Also cardiovascular patients have higher type $D$ personality than normal people $(P<0.01)$. In general, research findings indicate the importance of psychological factors associated with coronary artery disease.

\title{
Keywords
}

\author{
Quality of Family and Social Relationships, Type D Personality, Cardiovascular Disease
}

\section{Introduction}

The relationship between body and spirit has been noted and emphasized since $1000 \mathrm{BC}$.

Cardiovascular diseases (CVD) is caused by disorders of the heart and blood vessels, and includes coronary heart disease (heart attacks), cerebrovascular disease (stroke), raised blood pressure (hypertension), peripheral artery disease, rheumatic heart disease, congenital heart disease and heart failure.

Different biological factors, such as abnormal levels of certain fats in blood, increased cholesterol levels or decreased lipoprotein cholesterol density, decreased cholesterol levels or increased lipoprotein cholesterol, high blood pressure, smoking, lack of physical activity or exercise, being overweight or obese, increasing age, and family history of premature coronary heart disease make people susceptible to heart disease (Sher L, 2005) [1]. So far, many reasons have been mentioned for cardiovascular disease. Some physicians believe in genetic backgrounds and some point to other effects of physical illnesses on cardiovascular systems. However, numerous studies have shown that these factors alone do not play a decisive role in incidence of these diseases, but psychological and personality factors have a major impact on it (Rozanski, 2005) [2].

Personality characteristics play an important role in increasing or decreasing the negative effects of stress on cardiovascular performance. Contemporary evidence linking heart disease with psychological variables is drawn from the research that first was conducted by two cardiologists, Friedman and Rosenman (Rosenman RH, Brand RJ, Jenkins D, Friedman M, Straus R, Wurm M, 1975) [3].

According to the latest research findings, proceedings associated with prevention and also lifestyle play the greatest role in preventing cardiovascular diseases.

These findings are mainly based on these facts that facing urgent life events (such as job stress), repeated failure, marital conflicts, bad relationships with others and inappropriate social relationships increase the possibility of cardiovascular disease. These factors may be both the cause of the diseases and reasons involved in continuation or exacerbation of cardiovascular disease. Anger and aggression, being cynical, and educational, job or marital conflicts have negative effects on the cardiovascular system. Depression, acute anxiety, resentment, and anger can increase sodium in kidney, and thus cause cardiovascular dysfunctions.

Emotional traumas and abrupt pressures with increase in heart rate and secretion of some certain improper chemicals in blood increase the possibility of heart attacks and strokes.

Thus, identification of existing problems among people with coronary heart disease helps a lot to relieve physical and psychological damages. Since disorders and psychological conditions are useful in creation or exacerbation of the disease, it is necessary to examine this issue (Jaarsma, 2010) [4].

Among these factors the researcher would compare these two important variables in cardiac patients and normal subjects. The first variable is the quality of relationships in these patients.

The study by Lepore (1995) [5] on heart patients showed that lack of proper relationships, social support and expressing extreme hostility increase heart disease and associated mortality (Lepore SJ, 1995).

In this regard, Hoekstra et al. (2013) [6] in a review on quality of life and patients with heart disease relationship showed changes created by this disease affect on patient's satisfaction and quality of life, this means the restrictions make problem for job tasks, family, and social life of the patient and lead to social isolation and depression (Hoekstra, Tialda, Jaarsma, Tiny. Dirk J. van Veldhuisen, Hans L. Hillege, R Sanderman, 2013).

Personality characteristics may also have a profound impact on disease response procedures, compliance with condition, or patient's coping with the symptoms (Gustafsson, 2003) [7]. Mental state and personality of the person with heart disease make him/her susceptible to other illnesses. For example, the results of medical research in the Netherlands showed disorder and depressive symptoms increase stroke in patients with heart disease by two-fold (Khillan and Sidhu, 2009) [8]. 
In recent years a new personality structure has been proposed as the type D personality. This type is characterized through simultaneous tendency to experience negative emotions (negative feelings) and avoiding expressing these emotions in social interactions with others (social inhibition).Diagnosis of type D personality with measurement scales of the mentioned features is possible (Denollet, 2006) [9].

Also, many studies have shown that personality type is considered as a risk factor for various physical and psychological disorders. This personality type characterized by social prohibition and high negative emotions is often seen in patients with heart disease (Pelle, 2010) [10].

Habra et al. (2003) [11] in a study on type D personality and heart disease found that patients with heart disease with this type of personality simultaneously tend to experience negative emotions and emotional inhibition in social interactions and have no good relationships with others (Habra, M. E., Linden, W., 2003). Other researchers have also replicated and extended these results. For example, a study in Norway found that negative influence can predict mortality caused by heart disease (Habra et al., 2003) [11].

Barnett et al. (2009) [12] stated that patients with personality other than type D showed significant difference in perceived disease aspects. It is believed that type D condition is worse than other types.

Due to the increasing incidence and prevalence of heart disease and an increased emphasis of health and wellbeing researchers on relation and association of psychosocial factors with incidence and persistence of cardiovascular disease necessity to identify the factors and coping strategies inpatients, which is the objective of the present study, can be felt more than ever, and since including psychological interventions to change unhealthy behavior patterns, along with the main physical treatments in developing countries have led to a reduction of injuries and health improvement in these patients. For this reason, the present study has discussed psychological and social factors such as the quality of family and social relationships. Therefore, important implications are brought for prevention and treatment by determining how psychological factors may play a role in etiology of these diseases. Long-term effects of cardiovascular diseases, which severely affect personal, social, occupational, physical and mental health of patients, are significant both in patient's point of view due to serious concerns related to the disease and experts and researchers' point of view, whose main concern is accurate and scientific explanation of factors affecting the recovery of heart disease including psychological factors and achieving more effective strategies in order to prevent and treat the disease.

Thus, the main objective of this study is what difference exists in the quality of social, family relationships, and type D personality in patients with cardiovascular disease compared with non-patients.

\section{Materials and Methods}

This is a cross-sectional study.Statistical population involved cardiovascular patients who referred to the author's offices (both private and his office in Mazandaran Heart Center) from Jan 2011 till Dec 2012. The patient sample consisted of 150 volunteer patients selected targeted available. Patients, whose heart disease were confirmed by a cardiologist and suffered from no other chronic disease, were included. Features such as having at least primary education to complete the questionnaire and the condition of being married were taken into account. The patients were motivated to participate in the study and interview and provided necessary cooperation.

Then control group (normal people) was selected. Control group was chosen from individual visitors without the disease and were matched in terms of demographic characteristics (age, sex, marital status, and educational level) with patient group. Inclusion criteria included the absence of acute conditions such as the need for surgery, severe heart disorders, severe pain or fever, and also consenting to cooperate.

Pierce Quality relations and Personality Inventory (DS14) were completed explaining the study objectives. Demographic information was also obtained from patient by the physician. However, there were patients who returned incomplete questionnaires and for reasons such as physical fatigue and impatience refused to cooperate, therefore all of them were excluded and replaced by other patients. Collecting the questionnaires took more than 3 months due to prolonged questions and susceptible status of patients.

\section{Research Tools}

Questionnaires and interviews were used.

Pierce and Sarason's quality relations inventory (QEI) (2000) [13] was used to measure the quality of relationships, which measures interpersonal conflicts and the depth of their relationships in addition to perceived social support from parents, spouse, and friends. It contains 27 articles and has three subscales. Score is separately calculated for each scale. Thus, the questionnaire has 7 separated scores (support, conflict). Given three 
sources (parents, spouse, and friends), which each scale according to the number of questions and that each question is graded from 0 to 3 , the depth of relationship score is calculated. Then based on theoretical basis of tests, three independent average scores were obtained for measures of social support, and relationship conflict, and 7 independent average scores for parents, spouse, and friends support are achieved for conflicting relationships with parents, spouse, friends, and depth of relationships with parents, spouses, and friends. Scoring is calculated separately for each scale. Therefore, the questionnaire consists of 9 separate scores (support, conflict). Depth of the relationship is calculated considering three sources (parents, spouse, and friends) that each scale is graded according to the number of questions and each question is graded from 0 to 3 . Score is calculated. Then, based on the theoretical basis of the test three independent mean scores are achieved for scales of social support, conflict relationship, and depth of relationships; nine independent mean scores are achieved for support of parents, spouse and friends, conflicting relationships of parents, spouse and friends, and depth of relationships with parents, spouse and friends.

Cronbach's alpha coefficient for the social support, relationship depth and relationship conflict subscales were reported to be $0.83,0.88$ and 0.86 , respectively. This scale was calculated in 1992 in Iran and its reliability was 73\% (Ebrahimi, 1992). In this study, Cronbach's alpha coefficient for social support, relationship depth and relationship conflict subscales were obtained $0.89,0.86$ and 0.83 , respectively.

To assess type D personality, DS14 Questionnaire was used, which has been designed by Denolt and evaluates two general features including negative emotion and social inhibition. The questionnaire consisted of 14 questions, 7 of which are related to negative emotions and 7 other are related to social inhibition. Each subject answer to each question based on a 5-item scale (right-relatively right- nor right neither wrong-relatively wrongwrong). These options are respectively graded based on values of $0,1,2,3$, and 4 , where 0 means "never like man" and 4 means "always like me". The options are chosen, however grading in items 1 and 3 takes place reversely. Thus, for each subscale the subject's score ranges from 0 to 28 and for the entire scale it ranges from 0 to 56 .

Internal consistency of negative emotion subscale is 0.88 and social inhibition subscale is 0.86 (Denolt, 2005). Also, Cronbach's alpha coefficient has been reported to be 0.77 for negative emotion subscale, 0.69 for social inhibition subscale, and 0.79 for the whole scale (Zoljenahi, 2006). In the present study, alpha coefficient was obtained to be 0.80 for negative emotion subscale, 0.75 for social inhibition subscale, and 0.81 for the whole scale.

First observations were described by the help of descriptive statistics methods including tables, graphs, and indices of central tendency and distribution. Then, observations were described by inferential statistics methods such as univariate and multivariate analysis of variance and then were analyzed by SPSS.

\section{Results}

Descriptive findings: gender: Half of the subjects were male (150) and half were female (150).

Condition: Half of the samples (150) were healthy and half were patients with heart disease of the 300 participants, 108 patients (36\%) had the mean age of 52 to 63 years old and 30 patients (10\%) were 30 to 40 years old.

Descriptive findings of the study variables:

Table 1 includes descriptive parameters of the study variables by sex and education.

Table 2 includes descriptive parameters of the study variables by health status (condition).

Table 3 and Table 4 show the results of multivariate and univariate ANOVA.

The results listed in Table 3 show that $\mathrm{F}$ value all tests was significant in the health variable $(P<0.05)$, so the findings suggest that at least one dependent variable is the different between two healthy and ill groups. Review of individual variables would be followed in Table 4.

The results of Univariate ANOVA test show that there is a significant difference in three scales of social support, relationship conflict, and relation depth between normal people and patients with heart disease $(P<0.01)$. Comparison of mean values show that control group has achieved higher scores for social support and relation depth scales and lower scores for relationship conflict scale compared to patient group. So, the Cardiac Patients group has lower social support and depth of relationship and higher relationship conflict rather than normal population.

The results of multivariate and univariate ANOVA tests are shown in Table 5 and Table 6. 
Table 1. Descriptive indicators of the study variables by health status (Patient).

\begin{tabular}{|c|c|c|c|}
\hline \multicolumn{2}{|c|}{ Health status } & & \multirow{3}{*}{ Variable } \\
\hline \multicolumn{2}{|c|}{ Patient } & & \\
\hline$S D$ & $M$ & & \\
\hline 7.46 & 33.76 & Social Support & \multirow{3}{*}{ Quality Relation Questionnaire (QRI) } \\
\hline 10.20 & 69 & Relationship Conflict & \\
\hline 8.31 & 40.90 & Depth & \\
\hline 2.65 & 17.16 & Negative emotion & \multirow[b]{2}{*}{ Personality Scale (DS14) } \\
\hline 2.15 & 17.14 & Social inhibition & \\
\hline
\end{tabular}

Table 2. Descriptive indicators of the study variables by health status (Healthy).

\begin{tabular}{|c|c|c|c|}
\hline \multicolumn{2}{|l|}{ Health status } & & \\
\hline \multicolumn{2}{|c|}{ Healthy } & \multicolumn{2}{|c|}{ Variable } \\
\hline$S D$ & $M$ & & \\
\hline 3.71 & 46.01 & Social Support & \multirow{3}{*}{$\begin{array}{c}\text { Quality Relation } \\
\text { Questionnaire (QRI) }\end{array}$} \\
\hline 4.42 & 52.93 & Relationship Conflict & \\
\hline 5.05 & 54.62 & Depth & \\
\hline 2.85 & 7.98 & Negative emotion & \multirow{3}{*}{ Personality Scale (DS14) } \\
\hline & & & \\
\hline 3.12 & 8.27 & Social inhibition & \\
\hline
\end{tabular}

Table 3. Results of multivariate ANOVA to the dimensions of quality of relationships inventory (QRI) based on health status in the both healthy $(n=150)$ and heart disease $(n=150)$ groups.

\begin{tabular}{|cccc}
\hline \multicolumn{4}{|c}{ Health status (Healthy and ill) } \\
\hline The significance level & $F$ & Value of the statistic & Statistics \\
\hline 0.001 & 253.49 & 0.72 & Pillai's Trace \\
0.001 & 253.49 & 0.28 & Wilks' Lambda \\
0.001 & 253.49 & 2.57 & Hotelling's Trace \\
0.001 & 253.49 & 2.57 & Roy's Largest Root \\
\hline
\end{tabular}

Table 4. Results of univariate variance analysis related to dimensions of quality relationship inventory (QRI) based on health status in the both healthy $(n=150)$ and heart disease $(n=150)$ groups.

\begin{tabular}{cccccccc}
\hline \multicolumn{9}{c}{ Health status (Healthy or ill) } \\
\hline Square & The significance level & $F$ & Mean square & Degrees of freedom & Total square & Variable \\
\hline 0.52 & 0.001 & 323.72 & 11248.56 & 1 & 11248.56 & Social Support \\
0.51 & 0.001 & 313.02 & 19360.33 & 1 & $19360.33 \quad$ Relationship Conflict \\
0.50 & 0.001 & 297.94 & 14117.88 & 1 & 14117.88 & Depth \\
\hline
\end{tabular}


Table 5. Results of multivariate variance analysis related to the personality inventory (DS14) based on health status in the both healthy $(n=150)$ and heart disease $(n=150)$ groups.

\begin{tabular}{|cccc}
\hline \multicolumn{5}{c}{ Health status (Healthy or ill) } \\
\hline The significance level & $F$ & Value of the statistic & Statistics \\
\hline 0.001 & 564.55 & 0.79 & Pillai's Trace \\
0.001 & 564.55 & 0.21 & Wilks' Lambda \\
0.001 & 564.55 & 3.80 & Hotelling's Trace \\
0.001 & 564.55 & 3.80 & Roy's Largest Root \\
\hline
\end{tabular}

Table 6. Results of multivariate variance analysis related to the dimensions of Personality Inventory (DS14) based on health status in the both healthy $(n=150)$ and heart disease $(n=150)$ groups.

\begin{tabular}{cccccccc}
\hline \multicolumn{7}{c}{ Health status (Healthy or ill) } \\
\cline { 1 - 5 } Square & The significance level & $\boldsymbol{F}$ & Mean square & Degrees of freedom & Total square & Variable \\
\hline $\mathbf{0 . 7 4}$ & $\mathbf{0 . 0 0 1}$ & $\mathbf{8 3 3 . 2 2}$ & $\mathbf{6 3 2 9 . 6 1}$ & $\mathbf{1}$ & $\mathbf{6 3 2 9 . 6 1}$ & Negative emotion \\
$\mathbf{0 . 7 3}$ & $\mathbf{0 . 0 0 1}$ & $\mathbf{8 2 0 . 5 6}$ & $\mathbf{5 9 0 5 . 2 0}$ & $\mathbf{1}$ & $\mathbf{5 9 0 5 . 2 0}$ & Social inhibition \\
\hline
\end{tabular}

The results listed in Table 5 show that $\mathrm{F}$ values of healthy variables in all tests were significant $(P<0.05)$. So, at least a difference of one dependent variable in the two groups can be considered. Continuing review of each variable would be followed in Table 6.

\section{Discussion}

As noted in previous results, the study showed that the quality of family and social relationships were lower in cardiovascular patients compared to non-patients. These results are consistent with most previous studies. Atkinson (2000) [14] found that those who have problems and conflicts in their family and social relationships are one and half times more likely to have coronary heart disease than the others. Also this research showed that high family pressure along with stressful job may have a negative impact on cardiovascular health.

Lopez et al. (2008) [15] found that higher levels of functional support are associated with lower rates of arteriosclerosis (blockage of blood vessels). Doherty et al. (1983) [16] showed that social support from family and friends is associated with obedience and better adherence to medical recommendations.

To explain why the structural support is considered as a well-known predictor variable it can be noted that those with few friends or relatives usually have a higher mortality rate than those who have a higher level of structural support (Kaplan et al., 1994) [17].

Social support is one subscale of quality of relationships. Its significant difference between heart patients and non-patients suggests that structural support is a well-known predictor variable for mortality rate. Where do beneficial effects of social support come from? One possibility is that individuals with higher social support have higher health behaviors such as having a healthy diet, not smoking, and no alcohol consumption, and apply them more often. In addition, social support may have a direct impact on biological processes.

Studies in the framework of this theory have indicated that people with higher social support, whether they receive social support, compared to those with lower social support have more favorable health behaviors and follow them more. Conflict relations were another subscale of the quality of relationships and it was significantly different among heart patients and non-patients Participants. Fincham (2003) [18] in his review on this field concluded that conflicts influence on mental-physical health of families. He has proven the relationship between marital conflicts and depression, eating disorders, chronic diseases like cancer, and cardiovascular disease. Conflict in emotional relationships has impact on whole marital relationship. According to research, conflicting couples show the lowest level of satisfaction with marriage and the most divorce rate (Segrin, 2007) [19].

We can say Supporting role of families in these patients, especially of spouses in married ones, is very important. Spouses and families can be helpful by the emotional support, companionship, and understanding complications of the disease in these patients in order to improve quality aspects of life in these patients (Castro, 2012) [20]. 
During this study, it seemed those who had kind and supportive spouses or families showed higher comfort and mental health, and perceptions of the supports led to their healing process. Furthermore, anxiety and depression in these patients cause avoidance of expressing emotion in time and appropriately. To explain above findings, we can say family relationships in different parts are affected by the disease of one party. The incidence of disease changes the responsibilities and in some cases the roles of people, new circumstances are provided, and it is difficult for individuals to adapt with these conditions or accept the roles and responsibilities. However, the psychological consequences of the disease, such as anxiety, depression and stress can affect not only the patient but also other family members. Since social supports such as low-cost and appropriate treatment and employment insurance may not be available in all classes of a society, the situation could become exacerbated. It is difficult to diagnose disease in those with satisfactory and supporting marital relationships, and they will experience faster recovery if they become ill (Halford, 2003) [21].

Pedersen et al. (2006) [22] showed that social inhibition is a component of type D personality, and may increase the effects of negative emotions. It is indicated that patients had higher levels of social inhibition after myocardial infarction. These findings are consistent with previous ones, such as Denollet et al. (2003) [23] investigating type D personality and heart disease who found cardiovascular patients with this type of personality have simultaneous tendency to experience negative emotions and emotional inhibition in social interactions and have no good relationships with others.

The study revealed that after myocardial infarction in patients with high levels of social inhibitionn mortality was 4 times more than those with low levels of inhibition.

It also shows that cardiovascular patients always feel anger and have dual feelings due to many challenges in their relationships with others, and bear lots of pressure and discomfort. Thus, it is a ground for the risk of atherosclerosis and coronary heart disease in them.

Another subscale of quality of relationships was depth of the relationships, which showed a significant difference between heart patients and normal people. On the other hand, conflict leads to loss of intimacy of deep relationships, thus affects the sexual relationship.

Another explanation could be that physical disorders caused by the disease can lead to inability to work. Moreover, loss of income source leads to lack of funding their family life, which consequently leads to impaired family and social relationships.

To explain the above findings it can be said that family relationships is affected by disease of one of the parties in different aspects. Incidence of disease causes that responsibilities and in some cases the role of different people change and new conditions be provided, that compliance with these conditions or adapting the roles and responsibilities may be difficult. Besides, psychological consequences caused by disease such as anxiety, depression and stress affect not only the patient but also other family members. Since the social supports such as appropriate and low-cost treatments and job insurance may not be available in all society classes, it can also be effective in aggravating created conditions.

Other findings of this study showed that cardiovascular patients have more type D personality.

As the results of previous research and the present study show people with type D personality facing negative emotions, experience more confusion and restlessness, and this factor can increase psychosomatic illnesses for them.

From the clinical point of view, people with type D personality are prone to anxiety, tension, unhappiness, and pessimistic and negative view of life. Hiding emotion expressions in family environment increase depression in these patients (Mols, 2010) [24].

To explain this finding we can say, anxiety and depression in these patients make them avoid expressing appropriate and timely emotions. Avoiding express emotions can reduce the quality of interpersonal relationships and quality of sexual relationships in patients.

Another explanation emphasizes on character role in releasing Cortisol. Increased Cortisol may be mediator agent in relationships of type D personality. It can also have increasing risk of many mental and physical diseases. For example, Cortisol inhibits growth hormone and gonad axes. Reduction of growth hormone has relatively high risk for many disorders including premature cardiovascular disease in adults.

Unfavorable changes in the living conditions of family members caused by chronic illness lead to depression, anxiety and other mental disorders in family members. In difficult stages of the disease, families become tired and want these conditions be resolved as soon as possible. No doubt, withstanding such overwhelming circumstances makes family members face many mental problems (Ziabakhsh, 2013) [25]. 
Therefore, family members and caregivers play a crucial role in improving the patient's physical condition mental disorder prevention. Ensuring mental health of family members leads to mental health of patient and both parties will be protected from mental traumas.

It is recommended that in terms of using different methods in order to increase marital satisfaction and its impact on heart disease, further practical and scientific investments be conducted, since this disease is one of the first causes of mortality in the world and especially in our country.

Each study from the beginning, i.e. subject selection, to the implementation sections, analysis and conclusions has limitations. This research also has obstacles and limitations that are mentioned below:

- Limitations of the study population (sample) due to the specific physical conditions of patients, lack of cooperation in some patients

- Availability of the research sample

- Cross-sectional study, uncontrolled stress caused by hospitalization, lack of precise psychological control

- Using self report questionnaires

These factors provide limitations to generalize the findings, which should be considered and therefore results should be generalized with caution. It is recommended that in association with using different methods in order to increase marital satisfaction and its impact on cardiovascular disease, more practical and scientific investments should be done because this disease is in the first place of mortality in the world, especially in our country, Iran.

\section{Conclusions}

Given increasing this important disease, the variables of this study have preventive interventions in predicting this disease. With an emphasis on training computation skills in order to resolve interpersonal conflicts in the family, it is recommended to conduct timely and effective psychological interventions of psychological and personality assessment of patients during admitting to hospitals and clinics.

The findings include lack of information of the patients, family and society about the disease and how to deal with the patients, changes in family and social relationships of the patients due to physical symptoms caused by the disease, which have led to patients with cardiovascular problems experience issues including disorders in work and professional career, even in routine activities of life, disability to afford living expenses and basic needs of the family, disruption in family dynamics, and etc. These factors mention the need to design and implement a comprehensive care system for patients and their families, and guide us toward the culture of heart disease, physical and emotional support and training, and appropriate referrals to enjoy available and even global supportive resources.

\section{Recommendation}

In terms of methodology, it is recommended to select samples of different hospitals besides using longitudinal methods in future studies. It is also suggested to use outpatients in order to control stress caused by admission and accurate assessment of psychological status of patients before completing research tools. It is also recommended to conduct similar studies in other hospitals of the province and compare the results. This issue should be studied for other chronic diseases as well.

\section{References}

[1] Sher, L. (2005) Type D Personality: The Heart, Stress, and Cortisol. QJM, 98, 323-329. http://dx.doi.org/10.1093/qjmed/hci064

[2] Rozanski, A., Blumenthal, J.A. and Kaplan, J. (1999) Impact of Psychological Factors on the Pathogenesis of Cardiovascular Disease and Implications for Therapy. Circulation, 99, 2192-2217. http://dx.doi.org/10.1161/01.CIR.99.16.2192

[3] Rosenman, R.H., Brand, R.J., Jenkins, D., Friedman, M., Straus, R. and Wurm, M. (1975) Coronary Heart Disease in Western Collaborative Group Study. Final Follow-Up Experience of 8 1/2 Years. JAMA, 233, 872-892. http://dx.doi.org/10.1001/jama.1975.03260080034016

[4] Jaarsma, T., Steinke, E.E. and Gianotten, W.L. (2010) Sexual Problems in Cardiac Patients: How to Assess, When to Refer. The Journal of Cardiovascular Nursing, 25, 159-164. 
[5] Lepore, S.J. (1995) Cynicism, Social Support, and Cardiovascular Reactivity. Health Psychology, 14, 210-216. http://dx.doi.org/10.1037/0278-6133.14.3.210

[6] Hoekstra, T., Tiny, J., van Veldhuisen, D.J., Hillege, H.L., Sanderman, R. and Lesman-Leegte, I. (2013) Quality of Life and Survival in Patients With Heart Failure. European Journal of Heart Failure, 15, 94-102. http://dx.doi.org/10.1093/eurjhf/hfs148

[7] Gustavsson, J.P. (2003) The HP5 Inventory: Definition and Assessment of Five Health-Relevant Personality Traits from a Five-Factor Model Perspective. Personality and Individual Differences, 35, 69-89. http://dx.doi.org/10.1016/S0191-8869(02)00142-3

[8] Khillan, R., Sidhu, G.S. and Axiotis, C. (2009) Role of Fine Needle Aspiration (FNA) Cytology in Assessment of Cervical Lymphadenopathy. Journal of Clinical Oncology, 27, e19525.

[9] Denollet, J. (2006) DS14: Standard Assessment of Negative Affectivity, Social Inhibition, and Type D Personality. Psychosomatic Medicine, 67, 89-97. http://dx.doi.org/10.1097/01.psy.0000149256.81953.49

[10] Pelle, A.J., Schiffer, A.A., Smith, O.R., Widdershoven, J.W. and Denollet, J. (2010) Inadequate Consultation Behavior Modulates the Relationship between Type D Personality and Impaired Health Status in Chronic Heart Failure. International Journal of Cardiology, 142, 65-71.

[11] Habra, M.E., Anderson, J.C., Weinberg, J. and Linden, W (2003) Type D Personality Is Related to Cardiovascular and Neuroendocrine Reactivity to Acute Stress. Journal of Psychosomatic Research, 55, 235-245. http://dx.doi.org/10.1016/S0022-3999(02)00553-6

[12] Barnett, M.D., Ledoux, T., Garcini, L.M. and Baker, J. (2009) Type D Personality and Chronic Pain: Construct and Concurrent Validity of the DS14. Journal of Clinical Psychology in Medical Settings, 16, 194-199.

[13] Pierce, R.S., Frone, M.R., Russell, M., Cooper, M.L. and Mudar, P. (2000) A Longitudinal Model of Social Contact, Social Support, Depression, and Alcohol Use. Health Psychology, 19, 28-38. http://dx.doi.org/10.1037/0278-6133.19.1.28

[14] Atkinson, R. and Hilgard, H. (2009) Introduction of Psychology. Nolen, S., Barbara, H. and Wagenear, W.A., Eds., 15th Edition, Pat Bond.

[15] López-Martínez, Esteve-Zarazaga, R. and Ramírez-Maestre, C. (2008) Perceived Social Support and Coping Responses Are Independent Variables Explaining Pain Adjustment among Chronic Pain Patients. The Journal of Pain, 9, 373-379. http://dx.doi.org/10.1016/j.jpain.2007.12.002

[16] Doherty, W.J., Schrott, H.G., Metcalf, L. and Lasiello-Vailas, L. (1983) Effect of Spouse Support and Health Beliefs on Medication Adherence. Journal of Family Practice, 17, 837-841.

[17] Sadock, B.J. and Sadock, V.A. (2007) Kaplan and Sadock’s Synopsis of Psychiatry: Behavioral Sciences/Clinical.

[18] Fincham, F. (2003) Marital Conflict: Correlates, Structure, and Context. American Psychological Society, 12, 10.

[19] Segrin, C. and Taylor, M. (2007) Positive Interpersonal Relationships Mediate the Association between Social Skills and Psychological Well-Being. Personality and Individual Differences, 43, 637-646.

[20] Halford, W.K. (2003) Brief Therapy for Couples: Helping Partners Help Themselves. Guilford Press, New York.

[21] Costa, C.D., Sá, M.J. and Calheiros, J. (2012) The Effect of Social Support on the Quality of Life of Patients with Multiple Sclerosis. Arquivos de Neuro-Psiquiatria, 70, 108-113. http://dx.doi.org/10.1590/S0004-282X2012000200007

[22] Pedersen, S.S. and Denollet, J. (2006) Is Type D Personality Here to Stay? Emerging Evidence across Cardiovascular Disease Patient Groups. Current Cardiology Reviews, 2, 205-213. http://dx.doi.org/10.2174/157340306778019441

[23] Denollet, J., Conraads, V.M., Brutsaert, D.L., De Clerck, L.S., Stevens, W.J. and Vrints, C.J. (2003) Cytokines and Immune Activationin Systolic Heartin Systolic Heart Failure: The Role of Type D Personality. Brain, Behavior, and Immunity, 17, 304-309. http://dx.doi.org/10.1016/S0889-1591(03)00060-6

[24] Mols, F. and Denollet, J. (2010) Type D Personality among Noncardiovascular Patient Populations: A Systematic Review. General Hospital Psychiatry, 32, 66-72. http://dx.doi.org/10.1016/j.genhosppsych.2009.09.010

[25] Ziabakhsh, S. and Fazli, M. (2013) (CABG) in Hemodialysis-Dependent Patients and Comparison with Non-Renal Failure Patients. European Review for Medical and Pharmacological Sciences, 17, 2628-2631. 
Scientific Research Publishing (SCIRP) is one of the largest Open Access journal publishers. It is currently publishing more than 200 open access, online, peer-reviewed journals covering a wide range of academic disciplines. SCIRP serves the worldwide academic communities and contributes to the progress and application of science with its publication.

Other selected journals from SCIRP are listed as below. Submit your manuscript to us via either submit@scirp.org or Online Submission Portal.
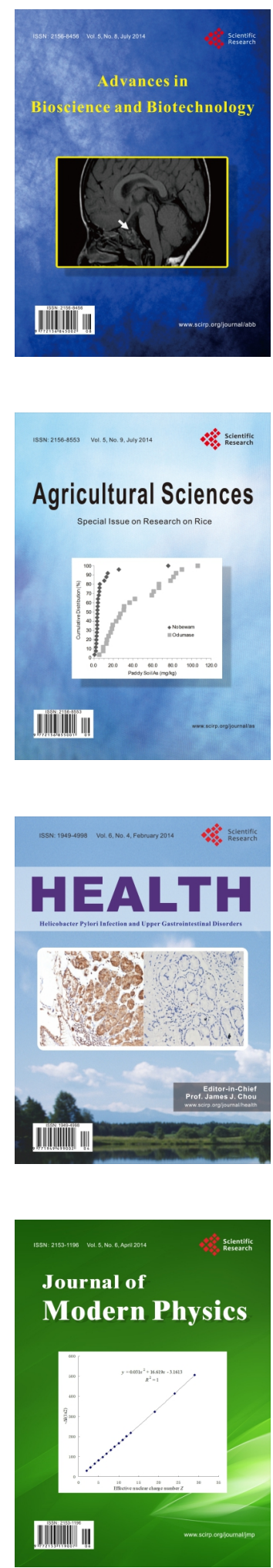
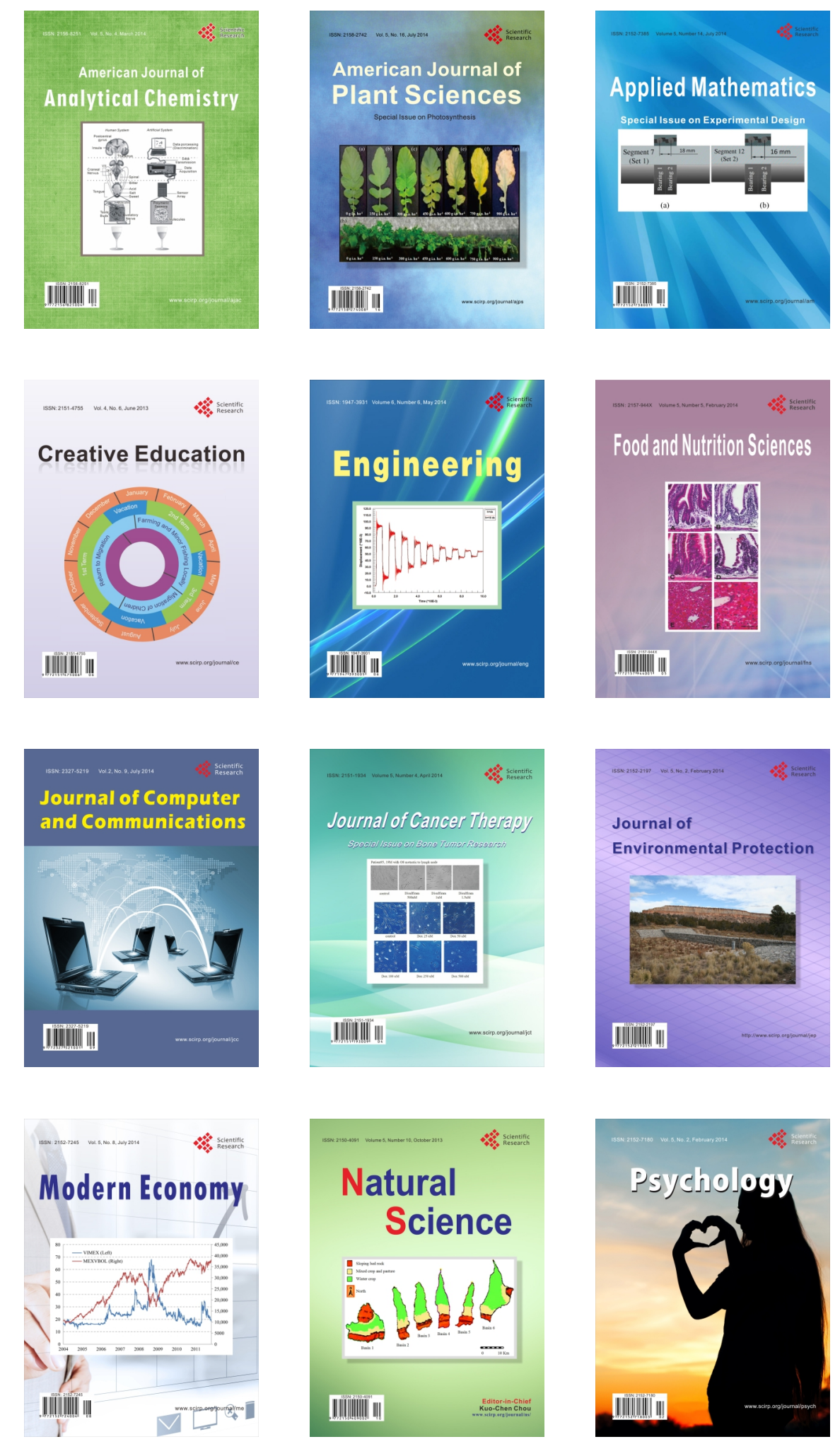